\title{
Strongly $n$-trivial Links are Boundary Links
}

\author{
Yukihiro TSUTSUMI \\ Sophia University \\ (Communicated by K. Taniyama)
}

\begin{abstract}
A link is said to be strongly $n$-trivial if there exists a diagram such that one can choose $n+1$ crossing points with the property that changing crossings on any $0<m \leq n+1$ points of these $n+1$ points yields a trivial link. It is shown that for a positive integer $n$ the components of a strongly $n$-trivial link admit mutually disjoint Seifert surfaces.
\end{abstract}

\section{Introduction}

Let $n$ be a non-negative integer. A knot (or link) $L$ in $S^{3}$ is said to be strongly $n$-trivial if there exists a diagram of $L$ such that one can choose $n+1$ crossing points with the property that changing crossings on any $0<m \leq n+1$ points of these $n+1$ points yields a trivial knot (or link). The collection of such crossing changes is called a strong $n$-trivializer for $L$. The link illustrated in Figure 1 is a non-trivial strongly 1-trivial link, where the strong 1-trivializer is indicated by dotted circles. H. Howards and J. Luecke [9] gave a construction of non-trivial strongly $n$-trivial knots for any given positive integer $n$ via "finger moves" [9, Section 6]. Strongly $n$-trivial links can be constructed as in Figure 2. Given a strongly $n$-trivial link, it is not so easy to detect the triviality of the link. Conversely, N. Askitas and E. Kalfagianni [1] showed that any strongly $n$-trivial knot is obtained from the unknot by "finger moves" on a Brunnian Suzuki graph.

It is well-known that any Vassiliev invariant of order $\leq n$ vanishes for strongly $n$-trivial knots. Further, Askitas and Kalfagianni [1] showed that if a knot $K$ is strongly $n$-trivial for $n \geq 2$, then $\nabla_{K}(z)=1$ [1, Theorem 1.2]. For a strongly 1-trivial knot $K$, it can be seen that $a_{2}(K)=0$ or \pm 1 , where $a_{2}(K)$ denotes the second coefficient of the Conway polynomial $\nabla_{K}(z)$, according to whether the trivializers are "unlinked" or "linked". This has been observed by T. Stanford (cf. [11]). Note that every strongly $n$-trivial knot has unknotting number one. It is well-known that every Alexander-Conway polynomial of knots is realized as that of unknotting number one knots (cf. [12], [13], [4], [19]).

Received January 30, 2006; revised May 25, 2006

Mathematics Subject Classification: 57M25.

Key words: strongly $n$-trivial link, boundary link.

Supported by Research Fellowships of the Japan Society for the Promotion of Science for Young Scientists. 


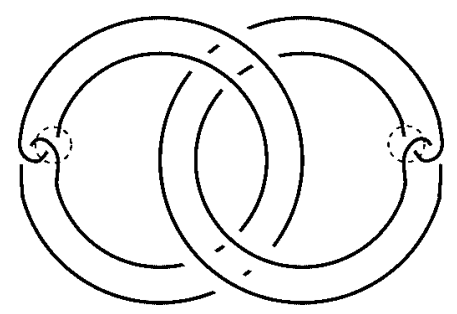

FIGURE 1. A strongly 1-trivial link.

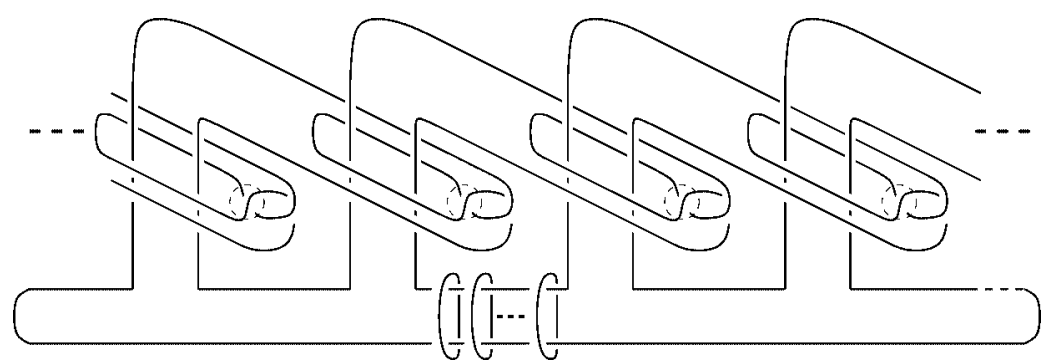

FIGURE 2

The strong $n$-triviality also gives some geometric restrictions. Several authors study minimal genus Seifert surfaces for strongly $n$-trivial knots by using techniques in 3 -dimensional topology. Howard and Luecke [9] showed that if $K$ is a non-trivial strongly $n$-trivial knot, then $n$ is less than $6 g(K)-3$, where $g(K)$ denotes the genus of $K$. In particular, the trivial knot is the only knot that is strongly $n$-trivial for all $n$ ([9, Corollary 1.3]). In [17] and [18], I. Torisu study strongly $n$-trivial 2-bridge knots and links.

THEOREM 1.1 ([17, Theorem 1.1]). A 2-bridge knot $K$ is strongly $n$-trivial for $n \geq 1$ if and only if $K$ is the trivial knot or the trefoil knot or the figure-eight knot.

THEOREM 1.2 ([18]). There is no non-trivial strongly $n$-trivial 2-bridge link for $n \geq$ 1.

In this note, we give some other geometric restriction to strongly $n$-trivial links. A link is called a boundary link if the components bound mutually disjoint Seifert surfaces. Note that a trivial link is a boundary link. It is well-known that if $L$ is a boundary link, then $\Delta_{L}(t)=0$. We also remark that the exterior $E(L)$ of a boundary link $L$ contains a closed incompressible surface which is not $\partial$-parallel (Proposition 4.3). Then, we show the following as a main result of this note.

THEOREM 1.3. Every strongly $n$-trivial link is a boundary link for $n \geq 1$.

As applications of Theorem 1.3 we have the following: 
COROLLARY 1.4. For a strongly $n$-trivial link $L, \Delta_{L}(t)=0$ for $n \geq 1$.

COROLLARY 1.5. The exterior of a non-splittable strongly $n$-trivial link contains a closed essential surface of positive genus for $n \geq 1$.

COROLLARY 1.6. For a non-trivial strongly $n$-trivial link $L$ with $\mu$ components, the rank of $\pi_{1}\left(S^{3}-L\right)$ is greater than or equal to $\mu+1$ for $n \geq 1$.

Recall that for a 2-bridge knot/link $L=S(a, b)$ in the Schubert form, $\Delta_{L}(-1)=a$ since the double branched covering space is the Lens space of type $(a, b)$. Recall also that the exterior of a 2-bridge link does not contain closed essential surfaces (cf. [7], [3]) and the rank of the fundamental group is two. Now we can reprove Theorem 1.2 by using any one of these facts together with Theorem 1.3.

\section{On Dehn surgery creating reducible 3-manifolds}

See [8] and [10] for basic terminology in 3-dimensional topology, and [14] for surgery, Seifert surfaces, boundary links. Throughout this paper, $N(\cdot)$ denotes the regular neighborhood and $E(\cdot)$ denotes the exterior, namely the complement of the interior of $N(\cdot)$. Unless stated otherwise, a link has at least two components. We consider Seifert surfaces for links $L$ as properly embedded surfaces $S$ in the link exteriors $E(L)$ such that for each component $T$ of $\partial E(L), T \cap S$ is a single circle intersecting a meridian exactly in one point.

We use the following results on non-trivial surgeries along a knot in reducible or $\partial$ reducible 3-manifolds that yield reducible or $\partial$-reducible 3-manifolds. In [16], M. Scharlemann studied surgeries producing reducible 3-manifolds. The following is a special case of his result.

THEOREM 2.1 (cf. [16, Theorem 6.1]). Let $M$ be a d-reducible 3-manifold. Let $K$ be a knot in $M$ such that the exterior $E(K)$ is irreducible and $\partial$-irreducible. If a non-trivial surgery along $K$ yields a reducible 3-manifold, then $K$ is a cable knot, and the surgery slope is that of the cabling annulus.

In particular, on Theorem 2.1 if a non-trivial surgery along $K$ yields a reducible 3manifold $M^{\prime}$, then $M^{\prime}$ is a Lens space summand. In [5], D. Gabai showed that if a non-trivial surgery along a knot $K$ in a solid torus yields a solid torus, then $K$ is 0 - or 1-bridge braid [5, Theorem 1.1 (1)]. The set of 1-bridge braids was classified in [6, Section 2]. In [2], J. Berge classified such knots and such non-trivial surgeries. See also [6, Section 3]. Then, we deduce the following lemma from [5, Theorem 1.1 (1)].

LEMmA 2.2. Let $K$ be a null-homologous knot in a solid torus $V$. If a non-trivial surgery along $K$ yields a solid torus, then $K$ bounds a disk in $V$.

We have the following lemma by using these results. 
LEMMA 2.3. Let $L_{0}$ be a trivial link in $S^{3}$. Let $\ell$ be a trivial knot in $S^{3}$ which is disjoint from $L_{0}$. Suppose that $\ell$ is null-homologous in $E\left(L_{0}\right)$. If the result of twisting $L_{0}$ along $\ell$ is a trivial link, then $\ell$ bounds a disk in $E\left(L_{0}\right)$.

Proof. Note that the exterior of a trivial link is reducible and $\partial$-reducible. Suppose that $E\left(L_{0} \cup \ell\right)$ is irreducible and $\partial$-irreducible. Then, by Theorem $2.1, \ell$ is a non-trivial cable about some knot in $E\left(L_{0}\right)$ and the surgery slope is given by the cabling annulus. This implies that the result of the surgery on $\ell$ is a Lens space summand, a contradiction since $H_{1}(E(L))$ is torsion free. Suppose that $E\left(L_{0} \cup \ell\right)$ is $\partial$-reducible. Then some component of $L_{0} \cup \ell$ bounds a disk whose interior is disjoint from $L_{0} \cup \ell$. If $\ell$ bounds a disk, we are done. Otherwise, some component of $L_{0}$ bounds a disk $D$, and $E\left(L_{0} \cup \ell\right)$ is reducible since $\partial N(D)$ is a separating sphere in $E\left(L_{0}\right)$ not bounding 3-balls. Now we may assume that $E\left(L_{0} \cup \ell\right)$ is reducible. Then there is a sphere $S$ in $E\left(L_{0} \cup \ell\right)$ which separates $S^{3}$ into two 3-balls $B_{1}, B_{2}$ such that $B_{i} \cap\left(L_{0} \cup \ell\right)$ is non-empty for $i=1,2, \ell \subset B_{1}$, and $E\left(B_{1} \cap\left(\ell \cup L_{0}\right)\right)$ is irreducible (cf. [8], [10]). The following lemma is easy to see.

LEMMA 2.4. If a link in a 3-ball is trivial in $S^{3}$, then the components of the link bound mutually disjoint disks in the 3-ball.

Thus, if $B_{1} \cap\left(L_{0} \cup \ell\right)=\ell$, then $\ell$ bounds a disk in $B_{1}$ by Lemma 2.4 since $\ell$ is a trivial knot in $S^{3}$. Suppose that $B_{1}$ contains exactly one component $k_{1}$ of $L_{0}$. Then each of $k_{1}$ and the result of twisting $k_{1}$ along $\ell$ is a trivial knot. Hence, by Lemma $2.2, \ell$ bounds a disk in $E\left(k_{1}\right)$, and in $B_{1}-k_{1}$ by Lemma 2.4 and the conclusion follows. Suppose that $B_{1}$ contains more than one components of $L_{0}$. Then the conclusion follows by Theorem 2.1 for the link $L_{0}^{\prime}=B_{1} \cap L_{0}$ in $S^{3}$ and $\ell^{\prime}=B_{1} \cap \ell$, since each of $L_{0}^{\prime}$ and the result of twisting $L_{0}^{\prime}$ along $\ell^{\prime}$ is trivial by Lemma 2.4. That is, $\ell^{\prime}$ bounds a disk in $B_{1}$, a conclusion for $L_{0}$ and $\ell$ as required. This completes the proof of Lemma 2.3.

\section{Proof of Theorem 1.3}

For convenience, we introduce $(n, m)$-triviality for links and prove the following:

THEOREM 3.1. For any positive integer $n$, each $(n+1, n)$-trivial link is a boundary link.

For positive integers $n$ and $m$ such that $n \geq m$, we say that a link $L$ is $(n, m)$-trivial if there is a diagram of $L$ such that one can choose $n$ crossing points with the property that for any integer $n^{\prime}$ with $n \geq n^{\prime} \geq m$, changing crossings of any $n^{\prime}$ points of the $n$ points yields a trivial link. Equivalently, for an $(n, m)$-trivial link $L$, there is a diagram of a trivial link $L_{0}$ which includes $n$ crossing points with the property that changing crossings on the $n$ points yields $L$ and for any integer $m^{\prime}$ with $n-m \geq m^{\prime} \geq 0$, chaining crossings on any $m^{\prime}$ points of the $n$ points yields a trivial link. Observe the following by definition:

PROPOSITION 3.2. (a) A link is strongly $n$-trivial if and only if it is $(n+1,1)$-trivial. 


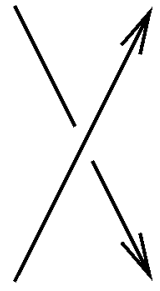

$L$

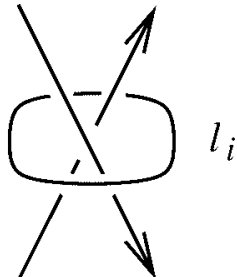

$L_{0}$

FIGURE 3. The -1 -surgery along $\ell_{i}$ changes $L_{0}$ into $L$.

(b) An $(n, m)$-trivial link is $(n, m+1)$-trivial for $n>m$.

Then we show that $(n+1, n)$-trivial links admit surgery descriptions as follows:

LEMMA 3.3. For an $(n+1, n)$-trivial $\mu$-component link $L=K_{1} \cup \cdots \cup K_{\mu}$, there is an $(n+\mu+1)$-component link $k_{1} \cup \cdots \cup k_{\mu} \cup \ell_{\mu+1} \cup \cdots \cup \ell_{n+\mu+1}$ such that $L_{0}=k_{1} \cup \cdots \cup k_{\mu}$ is a trivial link, $L$ is obtained from $L_{0}$ by suitable \pm 1 -surgeries along $\ell_{i}$ 's, and each $\ell_{i}$ bounds a disk in $E\left(L_{0}\right)$.

PROOF. Let $L_{0}$ denote the trivial link obtained from $L$ by the crossing changes on the $n+1$ crossing points. We put a circle $\ell_{i}$ near each of the $n+1$ crossing points as in Figure 3 so that $\operatorname{lk}\left(\ell_{i}, L_{0}\right)=0$. Then, we can recover $L$ from $L_{0}$ by the corresponding \pm 1 -surgeries along all $\ell_{i}$. Note that the link $L_{0}^{i}$ obtained from $L_{0}$ by performing a suitable \pm 1 -surgery along a component $\ell_{i}$ is a trivial link for $i=\mu+1, \ldots, n+\mu+1$ since $L$ is $(n+1, n)$-trivial. By the $(n+1, n)$-triviality of $L$ and counting the linking numbers, we see that each of the $n+1$ crossings is made by the same component of $L_{0}$, a "self-crossing". Thus, each $\ell_{i}$ is null-homologous in $E\left(L_{0}\right)$ since $\operatorname{lk}\left(\ell_{i}, L_{0}\right)=0$. Now by Lemma 2.3, we see that each $\ell_{i}$ bounds a disk in $E\left(L_{0}\right)$. This completes the proof.

LEMMA 3.4. Let $L_{0}=k_{1} \cup \cdots \cup k_{\mu}$ be a boundary link. Let $\ell_{\mu+1} \cup \cdots \cup \ell_{n+\mu+1}$ be a link in $E\left(L_{0}\right)$. Suppose that each $\ell_{i}$ bounds a disk in $E\left(L_{0}\right)$. Then $k_{1}, k_{2}, \ldots, k_{\mu}$ bound mutually disjoint Seifert surfaces disjoint from the $\ell_{i}$ 's.

PROOF. Let $S_{1}, S_{2}, \ldots, S_{\mu}$ be mutually disjoint Seifert surfaces for $k_{i}$ 's, and $D_{j}$ the disk bounded by $\ell_{j}$. We may assume that $S_{i}$ and $D_{j}$ are in general position. Then, $\left(S_{1} \cup \cdots \cup\right.$ $\left.S_{\mu}\right) \cap D_{j}$ consists of circles and proper arcs. If $\left(S_{1} \cup \cdots \cup S_{\mu}\right) \cap D_{j}$ is empty or consists of circles for each $j$, we are done. Suppose $\left(S_{1} \cup \cdots \cup S_{\mu}\right) \cap D_{j}$ has an arc component for some $j$, and let $\alpha$ be an outermost arc of $D_{j}$ with respect to the $\operatorname{arc}$ components. Let $\beta$ be the segment of $\ell_{j}$ corresponding to the outermost disk. Then, $\alpha$ is contained in some $S_{i}$ and $\beta$ is an arc such that the interior is disjoint from $S_{1} \cup \cdots \cup S_{\mu}$ and, since $\alpha$ is bi-collared in $D_{j}$, the ends of $\beta$ are on the same side of $S_{i}$. Attaching a tube to $S_{i}$ along $\beta$, we obtain a new system of disjoint Seifert surfaces $S_{i}^{\prime}$ for the components of $L_{0}$. This procedure reduces the number of arc components of $\left(S_{1} \cup \cdots \cup S_{\mu}\right) \cap D_{j}$ (and may produce circle components). Repeating 


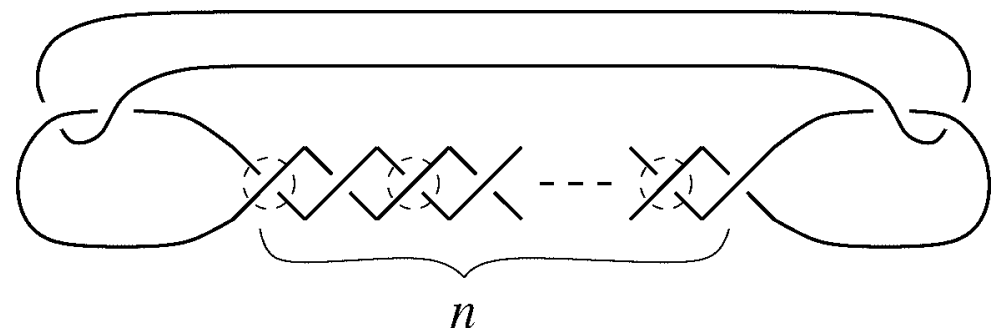

FIGURE 4. This link is not strongly $n$-trivial but $(n, n)$-trivial.

this procedure for $j=\mu+1, \mu+2, \ldots, n+\mu+1$, we get a desired system of disjoint Seifert surfaces. This completes the proof.

Now we are ready to prove Theorem 3.1.

Proof of Theorem 3.1. Let $L_{0}, \ell_{i}, k_{j}$ be as in Lemma 3.3. Then $L_{0}$ is a boundary link since it is a trivial link. By Lemma 3.4, there are mutually disjoint Seifert surfaces $S_{1}, \ldots, S_{\mu}$ for $k_{i}$ 's disjoint from any of $\ell_{i}$. Now $S_{j}$ 's become mutually disjoint Seifert surfaces $S_{i}^{*}$ 's for $K_{i}$ 's after corresponding \pm 1 -surgeries along $\ell_{i}$. This completes the proof of Theorem 3.1.

Proof of TheOREM 1.3. This follows from Proposition 3.2 and Theorem 3.1.

We remark that Theorem 3.1 (and the corollaries corresponding to Corollaries 1.4, 1.5, 1.6) are sharp in the sense that the link illustrated in Figure 4 is a 2-bridge $(n, n)$-trivial link but is not a boundary link.

The $(n, m)$-triviality gives a wider class than strongly $n$-trivial knots and links. Given a positive integer $n \geq 3$, let $n_{1}, n_{2}$ be positive integers such that $n-1=n_{1}+n_{2}$. Then for a strongly $n_{1}$-trivial knot $K_{1}$ and a strongly $n_{2}$-trivial knot $K_{2}$, it is easy to see that the composition $K=K_{1} \# K_{2}$, which is not an unknotting number one knot by Scharlemann [15], is an $(n+1, n)$-trivial knot but is not strongly $n$-trivial. Notice that if $L$ is unknotting number $n$, then $L$ is $(n, n)$-trivial. There are a plenty of links that are boundary links but not strongly $n$-trivial for any $n \geq 1$, that is, the opposite direction of Theorem 1.3 is not true. However, it appears unknown whether any boundary link is $(n, n-1)$-trivial for some $n$.

\section{Proofs of Corollaries}

It is well-known that $\Delta_{L}(t)=0$ for any boundary link $L$ and hence Corollary 1.4 follows. Corollary 1.5 follows from Theorem 1.3 and Lemma 4.3. Corollary 1.6 follows from Theorem 1.3 and Lemma 4.4.

LEMMA 4.1. The components of a $\mu$-component boundary link $L$ admit mutually disjoint Seifert surfaces $S_{1}, \ldots, S_{\mu}$ such that each $S_{i}$ is incompressible in $E(L)$. 
ProOf. We refer to $\sum g\left(S_{i}\right)$ as a complexity for the system $S_{1}, \ldots, S_{\mu}$ of mutually disjoint Seifert surfaces for the components of $L$. Let $S_{i}$ give the minimal complexity among disjoint Seifert surfaces. Then, we shall prove that, replacing $S_{i}$ 's if necessary by Seifert surfaces of the same complexity, each $S_{i}$ is incompressible in $E(L)$. If not, there is an embedded disk $D$ in $E(L)$ such that $D \cap S_{i}=\partial D$ and $\partial D$ does not bound a disk in $S_{i}$. We may assume that $D$ and the other $S_{j}$ 's are in general position and $D \cap\left(S_{1} \cup \cdots \cup S_{\mu}\right)$ is non-empty and consists of circles including $\partial D$. Let $D^{\prime}$ be an innermost disk of $D$ with respect to $D \cap\left(S_{1} \cup \cdots \cup S_{\mu}\right)$, and $S_{k}$ the component containing $\partial D^{\prime}$. If $\partial D^{\prime}$ does not bound a disk in $S_{k}$, then the compression of $S_{k}$ along $D^{\prime}$ yields the ones with smaller complexity, a contradiction. If $\partial D^{\prime}$ bounds a disk $D^{\prime \prime}$ in $S_{j}$, then replace $S_{j}$ with $S_{j}^{\prime}=\left(S_{j}-D^{\prime \prime}\right) \cup D^{\prime}$ to obtain a new system with the same complexity. Repeating this process finitely many times, we reach a contradiction to the minimality of the complexity. This completes the proof.

LEMMA 4.2. Let M be a compact, orientable, irreducible 3-manifold with non-empty boundary $\partial M$. If $\partial M$ is disconnected, then $M$ contains a closed incompressible surface of positive genus.

Proof. We construct disjoint surfaces $F_{i}$ in $M$ successively as follows: Start with a component $F_{0}$ of $\partial M$ and put $F_{1}=\partial N\left(F_{0}, M\right)-F_{0}$. Put $W_{1}=N\left(F_{0}, M\right)$. If $F_{i}$ has a sphere component $P$, then by the irreducibility $P$ bounds a 3-ball $C$ on the side not containing $W_{i}$ and regard $F_{i}-P$ as $F_{i}$ and $W_{i} \cup C$ as $W_{i}$. At this stage $F_{i}$ is not empty because $F_{i}$ separates $F_{0}$ from $\partial M-F_{0}$. Then, each component of $F_{i}$ has positive genus and $W_{i}^{\prime}=W_{1} \cup \cdots \cup W_{i}$ is a compression body in which $F_{i}$ is incompressible. If $F_{i}$ is incompressible in $M$, we stop. If $F_{i}$ is compressible in $M$, then it is compressible in $\operatorname{cl}\left(M-W_{i}^{\prime}\right)$ and we compress $F_{i}$ to obtain a new surface $F_{i+1}$ and write $W_{i+1}$ the compression body between $F_{i}$ and $F_{i+1}$. Since the complexity of $F_{i+1}$ is fewer than that of $F_{i}, F_{n}$ is incompressible for some $n$. Now it is elementary to show that each component of $F_{n}$ is incompressible in $M$.

LEMMA 4.3. The exterior of a non-splittable boundary link contains a closed essential surface of positive genus.

ProOF. A non-splittable boundary link $L$ with $\mu$-component $(\mu \geq 2)$ admits mutually disjoint incompressible Seifert surfaces $S_{1}, S_{2}, \ldots, S_{\mu}$ by Lemma 4.1. If $E\left(S_{1} \cup S_{2} \cup \ldots \cup S_{\mu}\right)$ has a reducing sphere $R$, then $R$ becomes a splitting sphere of $L$. Hence $E\left(S_{1} \cup S_{2} \cup \ldots \cup S_{\mu}\right)$ is irreducible. By Lemma 4.2, $E\left(S_{1} \cup S_{2} \cup \cdots \cup S_{\mu}\right)$ contains a closed incompressible surface $F$ of positive genus. By the incompressibility of $S_{i}$ and the irreducibility of $E\left(S_{1} \cup S_{2} \cup \ldots \cup S_{\mu}\right)$ we see that $F$ is incompressible in $E(L)$ by the standard innermost disk argument. If $F$ is parallel to a component of $\partial E(L)$, then $F$ bounds a solid torus $V$ in $S^{3}$ whose core is a component $k_{i}$ of $L$. However $k_{i}$ bounds the Seifert surface $S_{i}$ in $V$. This implies that $k_{i}$ is null-homologous in $V$ and cannot be a core, a contradiction. Hence $F$ is a desired one. This completes the proof. 
LEMMA 4.4. Let $L$ be a non-trivial $\mu$-component boundary link for $\mu>1$. Then the rank of $\pi_{1}\left(S^{3}-L\right)$ is greater than or equal to $\mu+1$.

Proof. Let $S_{1}, \ldots, S_{\mu}$ be disjoint Seifert surfaces for the components of $L$. Let $B_{\mu}$ be the bouquet of $\mu$ loops. Then there is a continuous map $f: E(L) \rightarrow B_{\mu}$ such that $f\left(E\left(S_{1} \cup \cdots \cup S_{\mu}\right)\right)$ is the vertex of $B_{\mu}$ and $f\left(N\left(S_{i}\right)\right)$ is the $i$ th loop of $B_{\mu}$. Notice that $f$ is a surjection which induces an epimorphism $f_{*}: \pi_{1}\left(S^{3}-L\right) \rightarrow F_{\mu}$, where $F_{\mu}$ is the free group of rank $\mu$. Therefore the rank of $\pi_{1}\left(S^{3}-L\right)$ is greater than or equal to $\mu$. If $\pi_{1}\left(S^{3}-L\right)$ is generated by exactly $\mu$ elements, then it can be shown that $f_{*}$ is injective since $F_{\mu}$ is the free group of rank $\mu$ and hence $\pi_{1}\left(S^{3}-L\right)$ is a free group. This implies that $L$ is trivial, a contradiction.

ACKNOWLEDGMENTS. The author would like to thank Ichiro Torisu for useful conversations.

\section{References}

[1] N. Askitas and E. Kalfagianni, On knot adjacency, Topology Appl. 126 (2002), 63-81.

[2] J. Berge, The knots in $D^{2} \times S^{1}$ which have nontrivial Dehn surgeries that yield $D^{2} \times S^{1}$, Topology Appl. 38 (1991), 1-19.

[3] W. FlOYD and A. HATCHER, The space of incompressible surfaces in a 2-bridge link complement, Trans. Amer. Math. Soc. 305 (1988), 575-599.

[ 4 ] H. FuJII, Geometric indices and the Alexander polynomial of a knot, Proceedings of the American Mathematical Society 124 (1996), 2923-2933.

[ 5 ] D. GabaI, Surgery on knots in solid tori, Topology 28 (1989), 1-6.

[6 ] D. GABAI, 1-Bridge braids in solid tori, Topology Appl., 37 (1990), 221-235.

[ 7 ] A. HATCher and W. ThuRston, Incompressible surfaces in 2-bridge knot complements, Invent. Math. 79 (1985), 225-246.

[ 8 ] J. Hempel, 3-Manifolds, Annals of Math. Studies 86, Princeton University Press.

[ 9 ] H. HowARDS and J. LueCKE, Strongly $n$-trivial knots, Bull. London Math. Soc. 34 (2002), 431-437.

[10] W. JACO, Lectures on Three-Manifold Topology, AMS. Conference board of Math. No. 43, 1977.

[11] E. Kalfagianni and X.-S. Lin, Knot adjacency and satellites, Topology Appl. 138 (2004) 207-217.

[12] H. KonDO, Knots of unknotting number 1 and their Alexander polynomials, Osaka J. Math. 16 (1979), no. 2, 551-559.

[13] H. MuraKami, Delta-unknotting number and the Conway polynomial, Kobe J. Math. 10 (1993), 17-22.

[14] D. Rolfsen, Knots and links, Publish or Perish, Inc, 1976

[15] M. Scharlemann, Unknotting number one knots are prime, Invent. Math. 82 (1985), no. 1, 37-55.

[16] M. Scharlemann, Producing reducible 3-manifolds by surgery on a knot, Topology 29 (1990), 481-500

[17] I. ToRisu, On strongly $n$-trivial 2-bridge knots, Math. Proc. Camb. Phil. Soc. 137 (2004), 613-616.

[18] I. ToRisu, Two-bridge links with strong triviality, Tokyo J. Math. 29 (2006), 233-237.

[19] Y. TsUTsumI and H. YAMADA, Variation of the Alexander-Conway polynomial under Dehn surgery, Topology 43 (2004), 893-901.

Present Address:

Department of Mathematics, Faculty of SCience and Technology, Sophia University, KIOICHO 7-1, CHIYODA-KU, TOKYO, 102-8554 JAPAN.

e-mail: tsutsumi@mm.sophia.ac.jp 